Memorias del VII Encuentro Nacional de Experiencias en la Enseñanza de la Biología y la Educación Ambiental y II Congreso Nacional de Investigación en la Enseñanza de la Biología

\title{
EL CONOCIMIENTO DIDÁCTICO DEL CONTENIDO 'DIVERSIDAD BIOLÓGICA': SU TRANSPOSICIÓN EDUCATIVA
}

\section{THE PEDAGOGICAL CONTENT KNOWLEDGE OF 'BIOLOGICAL DIVERSITY': ITS EDUCATIONAL TRANSPOSITION}

\author{
Gonzalo M. A. Bermúdez ${ }^{1}$
}

\section{Resumen}

Con el objetivo de evaluar el conocimiento de especies nativas de plantas y animales, el reconocimiento de componentes y atributos de la diversidad biológica, y la influencia de la gestión escolar y el género de los estudiantes en la mención de especies nativas en la provincia de Córdoba (Argentina), se tomó una cuestionario a una muestra de 338 alumnos/as de entre 15 y 18 años. También se analizó el concepto de biodiversidad desarrollado en 22 manuales escolares de los primeros tres cursos de la escuela secundaria de Argentina. Los resultados indican que los estudiantes poseen un buen conocimiento general de la flora y fauna autóctonas aunque fuera frecuente la mención de especies domésticas, ornamentales y adventicias como nativas. Además, la gestión escolar y el género de los estudiantes influyeron en el conocimiento de algunos vegetales y animales. Tanto los alumnos como los manuales escolares analizados se centraron en considerar el número de especies como componente y atributo principal de la biodiversidad. En base a estos resultados, concluimos que enseñar la diversidad biológica lleva implicados una serie de saberes docentes que se discuten en función del conocimiento didáctico del contenido.

Palabras Clave: Biodiversidad, conocimiento pedagógico del contenido, concepciones, manuales escolares, Argentina.

\section{Abstract}

In order to assess the knowledge of native plants and animals, the recognition of biodiversity components and attributes, and the influence of school management and gender to the mention native species in the province of Córdoba (Argentina), a questionnaire was taken to a sample of 338 students between 15 and 18 years old. The biodiversity concept developed in 22 textbooks of the first three high-school courses was also analyzed. The results indicate that students have a good general knowledge of the local flora and fauna, although it was common to mention

\footnotetext{
${ }^{1}$ Universidad Nacional de Córdoba, FCEFyN, Argentina. CONICET (Consejo Nacional de Investigaciones Científicas y Técnicas), Argentina. E-mail: gbermudez@com.uncor.edu
} 
Bio-grafía Escritos sobre la Biología y su Enseñanza.

Edición Extra-Ordinaria. ISSN 2027-1034 P. p 996 - 1007

Memorias del VII Encuentro Nacional de Experiencias en la Enseñanza de la

Biología y la Educación Ambiental y 11 Congreso Nacional de Investigación en la Enseñanza de La Biología

domestic, ornamental and adventitious species as native ones. In addition, school management and student gender influenced the knowledge of some plants and animals. Both students and textbooks focused on the number of species as the main attribute and component of biodiversity. Based on these findings we conclude that teaching biodiversity involve a number of teacher abilities which are discussed in the framework of the pedagogical content knowledge.

Keywords: Biodiversity, pedagogical content knowledge, conceptions, textbooks, Argentina.

\section{Introducción}

Cada disciplina tiene una dimensión didáctica inseparable de su contenido por lo que, tal como sostiene Acevedo Díaz (2009), resulta imprescindible cambiar la atención desde los enfoques más genéricos hacia otros más específicos de las asignaturas en la formación del profesorado. Esto reivindica la importancia de las didácticas específicas tanto en el reconocimiento de su estatus epistemológico (Adúriz-Bravo y Erduran, 2003) como de la necesidad de adecuar los currículos de los profesorados científicos.

En este contexto, Shulman (1986) denomina "Conocimiento Pedagógico del Contenido" (CPC) al "conocimiento que va más allá del tema de la materia per se y que llega a la dimensión del conocimiento del tema de la materia para la enseñanza" (p. 9). EI CPC incluye, por ejemplo, la representación y organización de los contenidos de enseñanza, su adaptación a los diversos intereses y habilidades de los estudiantes, el uso adecuado de analogías, ilustraciones y demostraciones útiles, etc. El CPC también ha sido traducido al castellano como Conocimiento Didáctico del Contenido (CDC), incorporando el entendimiento de las dificultades de aprendizaje de los alumnos, sus concepciones, intereses, redes de significado e, incluso, las formas de habla (Bermudez y De Longhi, 2012). Shulman (1986) diferenció inicialmente tres tipos de conocimientos del contenido en relación con la práctica educativa: el conocimiento de la materia a enseñar, el conocimiento pedagógico y el conocimiento del currículum. Más tarde, el mismo autor (Shulman, 1987) redefinió las categorías de saber y especificó que un profesor debería tener conocimiento de al menos siete dominios diferentes: a) conocimiento del contenido, b) didáctico general, c) curricular, d) didáctico del contenido, e) de los aspectos cognitivo-afectivos de los estudiantes, f) de los contextos educativos, y g) conocimiento de las finalidades y valores educativos.

Según Bermudez y De Longhi (2012), el CDC se centraría en los procesos de transposición y de comunicación ya que las situaciones didácticas ponen en juego la interacción entre docentes, alumnos y un determinado objeto de conocimiento. Este último, o saber "a enseñar", experimenta una serie de adecuaciones en su 
Bio-grafía Escritos sobre la Biología y su Enseñanza.

Edición Extra-Ordinaria. ISSN 2027-1034 P. p 996 - 1007

Memorias del VII Encuentro Nacional de Experiencias en la Enseñanza de la

Biología y la Educación Ambiental y 11 Congreso Nacional de Investigación en la Enseñanza de La Biología

modalidad y función desde su creación o descubrimiento hasta que llega a las aulas. Chevallard (1991) denominó a este proceso "transposición didáctica" y lo definió como el conjunto de modificaciones que experimenta un "objeto de saber" desde que se produce en la comunidad científica, pasando a su inclusión en el currículo oficial ("saber a enseñar") y, finalmente, hasta su planificación para la enseñanza ("objeto de enseñanza"). Años más tarde, Chevallard desarrolló una aproximación más antropológica a la transposición didáctica al incluir prácticas sociales de referencia como objetos de saber además de los conocimientos científicos (Gómez Mendoza, 2005).

Las investigaciones en Didáctica de las Ciencias han demostrado que muchas ideas de los alumnos son resistentes a los cambios, lo que puede significar un obstáculo para el proceso de aprendizaje. Algunas de las nociones sobre el contenido de la diversidad biológica (Díaz et al., 2006) son: (a) utilizar los términos "biodiversidad" y "número de especies" como sinónimos, dejando de lado otros componentes (genes, poblaciones, grupos funcionales, comunidades y unidades de paisaje) y atributos (abundancia relativa, rango, distribución espacial e interacciones) (Bermudez y De Longhi, 2008; Bermudez et al., en prensa); (b) reconocer y valorar especies principalmente animales $y$, en menor medida, vegetales de contextos cercanos, generalmente ricos en especies exóticas (Campos et al., 2012; Cardoso-Silva y de Oliveira, 2013; Schwarz et al., 2012); y (c) centrarse en "animales amigables" como los grandes mamíferos y en "plantas atractivas", con flores grandes, coloridas y perfumadas (Campos et al., 2012). En este marco, los objetivos del presente trabajo son evaluar (a) el conocimiento de especies nativas de plantas y animales, (b) el reconocimiento de componentes y atributos de la diversidad biológica, (c) la influencia de la gestión escolar (escuela pública o escuela privada) y el género (varón o mujer) en las especies nativas conocidas por estudiantes de nivel secundario de la provincia de Córdoba (Argentina); y (d) analizar el concepto de biodiversidad que se desarrolla en manuales escolares de los primeros tres cursos de la escuela secundaria de Argentina.

\section{Metodología}

\subsection{Cuestionarios a alumnos}

Se elaboró un cuestionario semi-estructurado que fue aplicado a un muestreo intencional de catorce cursos de escuela secundaria, correspondientes al ciclo de especialidad de escuelas públicas y privadas con orientación en Ciencias Naturales en la provincia de Córdoba (Argentina), con un total de 338 alumnos encuestados (15-18 años). Se pidió a los estudiantes que completaran una lista con diez nombres de plantas y diez de animales nativos. También se les solicitó que determinaran en una serie de esquemas ambientales cuál tenía mayor 
Memorias del VII Encuentro Nacional de Experiencias en la Enseñanza de la

Biología y la Educación Ambiental y 11 Congreso Nacional de Investigación en la

\section{Enseñanza de la Biología}

biodiversidad para crear allí una reserva biológica. Para su confección se utilizaron figuras de árboles, arbustos, hierbas y cactus (Césare et al., 2013), que combinadas en distinto número y criterio de agrupamiento representaban diferentes componentes y atributos de la diversidad biológica (Díaz et al., 2006) (Anexo 1). Se identificaron los nombres científicos, otros vernáculos y el estatus de las especies animales y vegetales mencionadas en el cuestionario.

Para las animales, se categorizaron los nombres dados en: especie nativa (original de Argentina), exótica (original de otros países), introducida (especie exótica que vive en Argentina), mixta (nombre genérico que contiene especies tanto nativas como exóticas) y doméstica (mascotas y animales de granja). En tanto que las categorías de plantas fueron: especie nativa, exótica, mixta y adventicia (especies exóticas que se propagan espontáneamente en Argentina). Para el análisis estadístico se realizaron pruebas Chi-cuadrado $\left(X^{2}\right)$ para tablas de contingencia (datos categorizados) y Modelos Lineales Generalizados Mixtos (MLGM) (datos continuos) con el programa Infostat (Universidad Nacional de Córdoba, versión 2012). Estos últimos surgieron de cuantificar el número de especies que cada alumno mencionó (con un mínimo de cero y un máximo de diez), el número de especies nativas, exóticas, mixtas, adventicias y domésticas (según las categorías correspondan a animales o plantas). Para el análisis MLGM, los cursos fueron considerados como efectos aleatorios, a la vez que la gestión escolar y género de los estudiantes como efectos fijos (con interacción). La distribución fue binomial con una función de identidad de vínculo de tipo "Logit". El procedimiento general de análisis y procesamiento de los datos fue realizado según lo indicado por Bermudez et al. (2012) y Campos et al. (2012).

\subsection{Libros de texto}

Se utilizó como herramienta de trabajo una grilla adaptada de la ya validada por Bermudez et al. (en prensa) para manuales de España. Se registró como variable respuesta la presencia explicita, implícita y ausencia del tratamiento del contenido especificado de cada ítem del instrumento. La presencia implícita fue definida en este trabajo para ocasiones en las que un tema particular se desarrollara fuera del tratamiento conceptual específico de la biodiversidad. Las editoriales argentinas analizadas fueron: Aique Grupo Editor, Estrada, Kapeluz, Longseller, Maupie, Puerto de Palos, Santillana, SM, Thompson Learning y Tinta Fresca $(n=22$ manuales). El tratamiento estadístico se realizó mediante la prueba de prueba $\mathrm{x}^{2} \mathrm{y}$ los resultados se expresaron como porcentajes. Se realizó además un Análisis de Componentes Principales (ACP) para determinar un nuevo conjunto de variables que nos permitan distinguir aquellos libros de texto que presenten la conceptualización de biodiversidad más completa (en cuanto al número de componentes y atributos tratados) y compleja (relaciones establecidas entre los mismos). 
Bio-grafia Escritos sobre la Biologia y su Enseñanza.

Edición Extra-Ordinaria. ISSN 2027-1034 P.p 996 - 1007

Memorias del VII Encuentro Nacional de Experiencias en la Enseñanza de la

Biología y la Educación Ambiental y II Congreso Nacional de Investigación en la Enseñanza de la Biología

\section{Resultados y Discusión}

\subsection{Nociones de la biodiversidad de alumnos de escuela secundaria}

\subsubsection{Especies animales y vegetales nativas}

Los alumnos de escuela secundaria de la provincia de Córdoba (Argentina) nombraron 217 especies de animales, siendo las más frecuentes el puma, el cóndor, la liebre, el gato montés, la vizcacha, el zorro, el quirquincho, el cuis, la vaca y la yarará (Figura 1). Sin embargo, si consideramos los diez espacios brindados a los estudiantes para completar el listado de especies nativas (338 alumnos $\times 10$ respuestas posibles $=3380$ respuestas totales), la ausencia de respuesta fue la categoría más frecuente $(n=659)$. En este sentido, menos de la mitad de los alumnos (46.0\%) nombró las diez especies animales solicitadas. Los resultados del presente estudio están en desacuerdo con los publicados por Campos et al. (2012), quienes encontraron que animales domésticos (como el perro y el gato) y exóticos (como el león) fueron los más elegidos por niños y adolescentes. En relación con las especies vegetales, el algarrobo fue la especie más mencionada, seguida por el espinillo, el sauce, el pino, el piquillín y el siempre verde (Figura 1). El resto de las 145 especies mencionadas tuvieron una frecuencia menor al 3\%. Como en el caso de las especies animales, la ausencia de respuesta fue la categoría más frecuente (33.6\%).

El estatus de las especies animales más frecuente fue la categoría nativa $(65.2 \%$, Tabla 1), lo que se encuentra en desacuerdo con los resultados del estudio realizado por Nates et al. (2010) en Argentina. A su vez, la gestión escolar y el sexo de los estudiantes resultaron variables que influyeron en el estatus de algunas de las especies mencionadas (prueba $\mathrm{X}^{2}$ ). Por ejemplo, de las 217 especies animales nombradas, la frecuencia de mención de 26 especies fue significativamente diferente según la gestión de la institución escolar o el sexo de los estudiantes (Tabla 1). En el caso de las escuelas de gestión privada, los alumnos varones nombraron con mayor frecuencia al puma, cóndor, zorro, cuis, yarará, guanaco, ñandú, perdiz, llama y peludo (datos no mostrados), mientras que las mujeres mencionaron a la mayoría de los animales nativos: puma, cóndor, zorro, yarará, guanaco, ñandú, perdiz, llama, peludo (datos no mostrados). Por otro lado, los estudiantes de escuelas públicas nombraron más frecuentemente a la vaca, el pato, el caballo, el perro, el burro, la garza, la paloma, el gato, la iguana, la gallina, el zorzal, la carpa, la liebre mara, el chancho del monte, la carpa, el hornero y el ganso (datos no mostrados). Además, las mujeres asistentes a escuelas de este tipo de gestión mencionaron más frecuentemente al caballo, perro, burro, garza, paloma, gato, gallina, zorzal, liebre mara y ganso (datos no mostrados). Estos resultados indican que factores asociados a la gestión escolar, como las características socio-económicas de los alumnos (Cervini, 2003), el tipo de 
Bio-grafía Escritos sobre La Biología y su Enseñanza.

Edición Extra-Ordinaria. ISSN 2027-1034 P. p 996 - 1007

Memorias del VII Encuentro Nacional de Experiencias en la Enseñanza de la

Biología y la Educación Ambiental y II Congreso Nacional de Investigación en la Enseñanza de la Biología

actividades que llevan a cabo y los ambientes en los que las realizan, aspectos a su vez atravesados por estereotipos y expectativas de género, influyen en las especies animales nativas de referencia de los estudiantes.
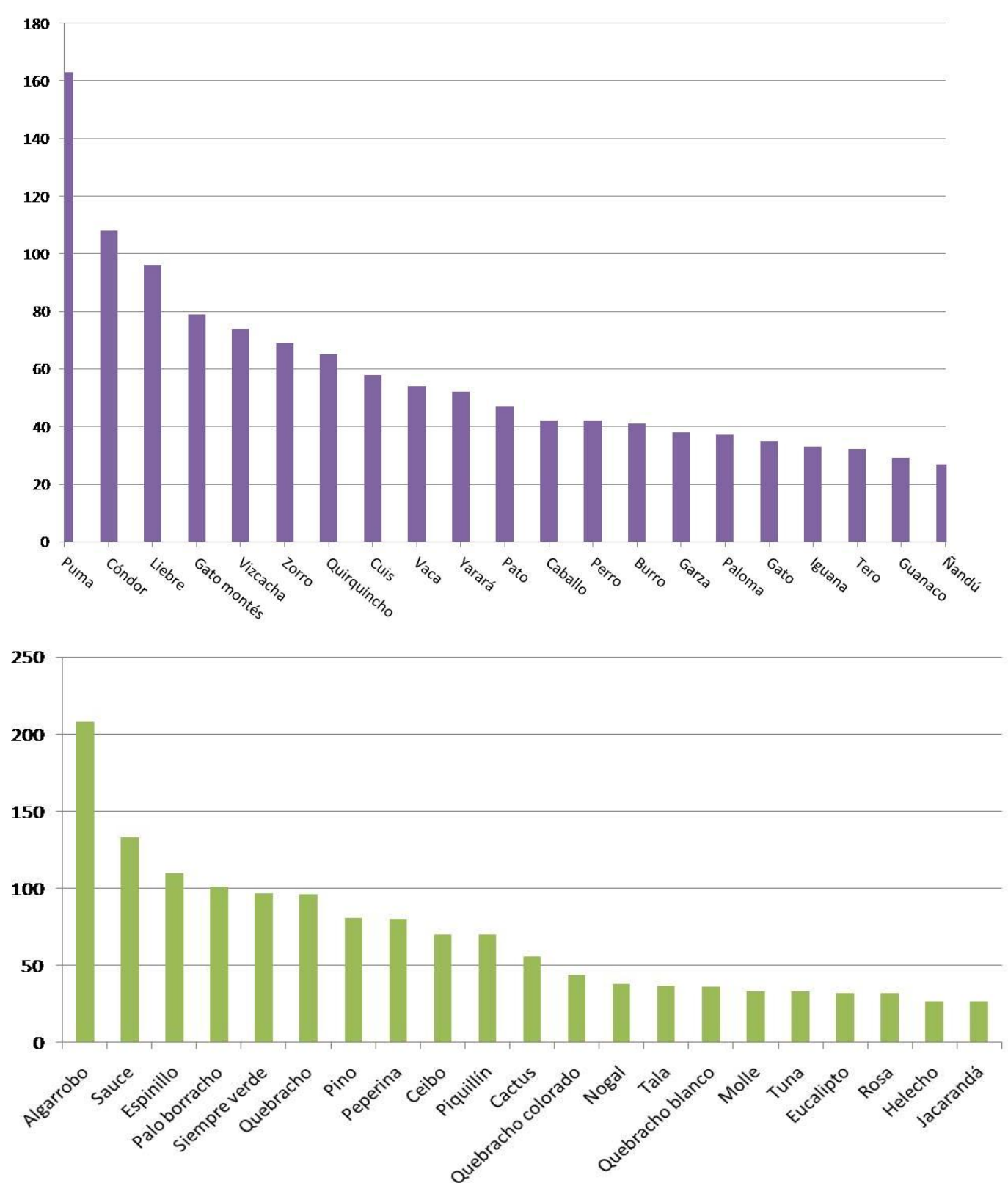

Figura 1. Frecuencia absoluta del número de menciones de especies dados por estudiantes de escuela secundaria de la provincia de Córdoba $(n=338$ alumnos). Arriba: especies animales $(p<0.001)$. Abajo: especies vegetales $(p<0.001)$. 
Memorias del VII Encuentro Nacional de Experiencias en la Enseñanza de la

Biología y la Educación Ambiental y 11 Congreso Nacional de Investigación en la Enseñanza de la Biología

En cuanto a las plantas, los estudiantes que asisten a escuelas públicas mencionaron más especies y, dentro de éstas, a un mayor número de nativas que los estudiantes de escuelas privadas (datos no mostrados). La tendencia a nombrar más especies nativas por estudiantes de escuelas públicas también fue significativa para las tres y cinco primeras especies mencionadas $(p<0.001)$. Estos resultados podrían explicarse por dos razones: (a) que las escuelas públicas estudiadas fueron más efectivas que las escuelas privadas en la promoción del aprendizaje de la flora argentina, o (b) que los adolescentes que asisten a escuelas públicas tienen un conocimiento ecológico más profundo debido a su estilo e historias de vida (Campos et al., 2012).

\subsubsection{Componentes y atributos de la biodiversidad}

La mayoría de los estudiantes no pudo reconocer la riqueza de individuos de una especie o de un grupo funcional en un ambiente de mayor biodiversidad que otro (Tabla 2). Por ejemplo, resulta idéntico para los alumnos un ambiente con tres especies y tres individuos por especie que uno con las mismas especies, pero con sólo un individuo de cada una. Estos hallazgos ponen de manifiesto la falta de conocimiento acerca de la ecología de la conservación y la genética de poblaciones, ya que por cuestiones de tamaño efectivo resultaría inviable un ecosistema con tan solo un representante de cada especie. Estas respuestas son consideradas que forman parte de un modelo de conservación que denominamos "arca de Noé". Por otro lado, en ambientes en los que varía la riqueza de grupos funcionales (manteniendo constante el número de especies, número de individuos totales y por especie), la mayoría de los estudiantes reconoció la opción con mayor biodiversidad $(55.9 \%$ ). En cuanto al rango (diferencia entre el valor máximo y valor mínimo) dentro de un grupo funcional (forma de la copa de árboles), la mayoría de los alumnos respondió que cualquiera de los dos ambientes representa la misma biodiversidad (51.1\%), ya que no reconocieron las diferencias en la estructura del dosel y de micro-hábitats para especies que pueden refugiarse en, o alimentarse de, los árboles graficados (Tabla 2).

\begin{tabular}{|c|c|c|c|c|c|}
\hline \multirow{3}{*}{$\begin{array}{l}\text { Estatus de las especies } \\
\text { animales }\end{array}$} & \multirow{3}{*}{$\begin{array}{l}\text { Frecuencia } \\
(\%)\end{array}$} & \multicolumn{4}{|c|}{ Gestión escolar (\%) } \\
\hline & & \multicolumn{2}{|c|}{ Privada } & \multicolumn{2}{|c|}{ Pública } \\
\hline & & Mujer & Varón & Mujer & Varón \\
\hline Doméstica & 16.46 & 2.34 & 1.95 & 7.58 & 4.59 \\
\hline Exótica & 2.21 & 0.35 & 0.48 & 0.69 & 0.69 \\
\hline Introducida & 7.11 & 1.73 & 1.69 & 1.91 & 1.78 \\
\hline Mixta & 9.01 & 1.56 & 1.78 & 3.55 & 2.12 \\
\hline Nativa & 65.21 & 18.07 & 14.34 & 17.76 & 15.03 \\
\hline Total & 100 & 24.05 & 20.23 & 31.5 & 24.22 \\
\hline Chi-cuadrado & $p<0.001$ & \multicolumn{4}{|c|}{$<0.001$} \\
\hline
\end{tabular}


Memorias del VII Encuentro Nacional de Experiencias en la Enseñanza de la

Biología y la Educación Ambiental y 11 Congreso Nacional de Investigación en la Enseñanza de la Biología

Tabla 1. Distribución de frecuencias y pruebas Chi-cuadrado para el estatus de las especies animales mencionadas (para Argentina), sin clasificar y clasificando por tipo de gestión institucional y sexo de alumnos de escuela secundaria de Córdoba.

La Tabla 2 también muestra que los estudiantes no reconocieron la equitatividad de las especies en los ambientes graficados, ya que la mayoría (52.3\%) respondió que representa la misma biodiversidad un ambiente con los individuos homogéneamente repartidos entre las especies que otro en el que se encuentra una especie dominante y el resto son especies con baja representatividad. Resultados similares fueron encontrados para la distribución espacial de especies, en donde el $51.7 \%$ de los alumnos reconoció como idénticos dos ambientes en los que las especies tienen una distribución aleatoria frente a otro donde se distribuyen de forma agregada y homogénea. En relación con las interacciones entre especies, la mayoría de los alumnos falló en diferenciar redes tróficas con las mismas especies pero con distinto número relaciones alimentarias entre las mismas (38.7\%, Tabla 2). Por el contrario, el $47.7 \%$ de los estudiantes reconoció que la biodiversidad aumenta con el número de unidades de paisajes en una región.

\subsection{Nociones de la biodiversidad presentes en manuales escolares}

\begin{tabular}{llllllll}
\hline $\begin{array}{l}\text { Componente } \\
\text { y atributo } \\
\text { Respuesta }\end{array}$ & $\begin{array}{l}\text { Riqueza } \\
\text { de } \\
\text { individuos }\end{array}$ & $\begin{array}{l}\text { Riqueza } \\
\text { de grupos } \\
\text { fun- } \\
\text { cionales } \\
\text { (GF) }\end{array}$ & $\begin{array}{l}\text { Equita- } \\
\text { tividad } \\
\text { de es- } \\
\text { pecies }\end{array}$ & $\begin{array}{l}\text { Distribución } \\
\text { espacial de } \\
\text { especies }\end{array}$ & $\begin{array}{l}\text { Rango } \\
\text { dentro } \\
\text { de un } \\
\text { GF }\end{array}$ & $\begin{array}{l}\text { Interac- } \\
\text { ciones } \\
\text { entre } \\
\text { especies }\end{array}$ & $\begin{array}{l}\text { Unida- } \\
\text { des de } \\
\text { paisaje }\end{array}$ \\
\hline $\begin{array}{l}\text { Reconoce } \\
\text { a }\end{array}$ & 40.2 & 55.9 & 20.5 & 32.3 & 23.6 & 33.8 & 47.7 \\
$\begin{array}{l}\text { No reconoce } \\
\text { b }\end{array}$ & 1.5 & 13.3 & 8.8 & 3.6 & 3.6 & 8.2 & 30.2 \\
$\begin{array}{l}\text { Confunde } \\
\text { c }\end{array}$ & 46.5 & 17.5 & 52.3 & 51.7 & 51.1 & 38.7 & 8.2 \\
$\begin{array}{l}\text { No relaciona } \\
\text { d }\end{array}$ & 3.3 & 5.1 & 7.9 & 3.9 & 10.9 & 9.1 & 4.2 \\
No responde & 8.5 & 8.2 & 10.6 & 8.5 & 10.9 & 10.3 & 9.7 \\
$P$ & $<0.001$ & $<0.001$ & $<0.001$ & $<0.001$ & $<0.001$ & $<0.001$ & $<0.001$ \\
\hline
\end{tabular}

Si bien el concepto de diversidad biológica estuvo presente en la mayoría de los libros de texto analizados, la definición se circunscribió, en general, al componente específico. Sólo el $34 \%$ de los manuales presentó una conceptualización que abarcara a las especies, los genotipos o poblaciones y a los ecosistemas ("trilogía de la biodiversidad") (datos no mostrados). En este mismo sentido, conceptos como las unidades de paisaje, y atributos como el rango y la distribución espacial estuvieron completamente ausentes. Estos resultados son similares a los hallados para los manuales de España de un nivel de escolaridad equivalente al EGB3 
Bio-grafía Escritos sobre la Biología y su Enseñanza.

Edición Extra-Ordinaria. ISSN 2027-1034 P. p 996 - 1007

Memorias del VII Encuentro Nacional de Experiencias en la Enseñanza de la

Biología y la Educación Ambiental y 11 Congreso Nacional de Investigación en la Enseñanza de La Biología

(Bermudez et al., en prensa). El agrupamiento de las variables que arrojó el ACP (datos no mostrados) señala que los componentes de la biodiversidad más correlacionados con su conceptualización fueron las especies, las poblaciones y las comunidades. Por otro lado, la riqueza, los grupos funcionales y las interacciones fueron variables con un peso menor en el porcentaje de variación explicada (correlacionadas con el Componente 2). Además, se encontró un amplio rango en la distribución de los manuales a lo largo del Componente 1, siendo los libros identificados con los números 22, 4, 2, 9, 12 y 14 los mayormente relacionados con las variables analizadas. Los valores de los autovalores que superaron la unidad fueron los correspondientes a los Componentes 1 (3.34), 2 (1.48) y 3 (1.28).

Tabla 2. Porcentaje de componentes y atributos de la diversidad biológica que reconocen estudiantes de escuela secundaria de Córdoba (Argentina) en ambientes esquemáticos. $\mathrm{P}=$ Valor de $\mathrm{p}$ de la prueba Chi-cuadrado. ${ }^{\mathrm{a}}$ Escoge el gráfico con mayor biodiversidad para el componente y atributo representado. ${ }^{b}$ Elige ambiente de menor biodiversidad. ${ }^{c}$ Identifica que la biodiversidad es idéntica en los dos ambientes mostrados. ${ }^{d}$ No considera que lo graficado tenga relación con la biodiversidad.

\section{Conclusiones}

En relación con el conocimiento de especies nativas tanto vegetales como animales, los estudiantes de escuela secundaria de Córdoba (Argentina) poseen un buen conocimiento general de la flora y fauna autóctonas. Sin embargo, los alumnos incluyeron en la categoría "nativa" a especies domésticas (como el perro, el gato, el caballo, etc.), ornamentales (como la rosa, el helecho, etc.) y, principalmente, las naturalizadas; es decir, las especies exóticas que se reproducen espontáneamente en un área fuera de su rango de distribución original (como el siempre verde, el pino, la liebre, etc.). Tanto la gestión escolar como el género de los estudiantes influyeron en el conocimiento de algunos vegetales, animales y, principalmente, en las categorías de especies nativas y exóticas. Estas cuestiones sugieren el tratamiento específico de los factores socioeconómicos que influyen en la asistencia a escuelas públicas o privadas y a los estereotipos de género que intervienen en las actividades que realizan varones y mujeres con la diversidad biológica. En cuanto a los componentes y atributos, los alumnos se centraron en considerar el número de especies como componentes y atributos principales de la biodiversidad. No obstante, la mayoría de los estudiantes reconoció dos aspectos importantes como son la riqueza de grupos funcionales y de unidades de paisaje. Estos resultados indican que se vuelve necesaria la implementación de estrategias didácticas destinadas a ampliar las concepciones de la biodiversidad y de incorporar componentes y atributos que, si bien generalmente no son tenidos en cuenta en la enseñanza, llegan a ser 
Memorias del VII Encuentro Nacional de Experiencias en la Enseñanza de la Biología y la Educación Ambiental y II Congreso Nacional de Investigación en la

\section{Enseñanza de la Biología}

correctamente identificados por los alumnos. Por otro lado, los manuales escolares siguen una tendencia a representar el concepto de biodiversidad con el de diversidad específica, lo que influye negativamente en el proceso de transposición didáctica. Por esta razón invitamos al profesorado a analizar cuidadosamente los materiales para el trabajo en el aula, vigilando la epistemología del contenido, identificando tanto los errores conceptuales como las visiones desactualizadas de la actividad científica. Trabajar "en la transposición" implica seleccionar, organizar y secuenciar contenidos como el de la biodiversidad, revisar la disciplina y elegir un enfoque que le otorgue a la propuesta de clase (objeto de enseñanza) un sentido ecológico y social. Lo anterior convierte al docente en un mediador calificado en la construcción del conocimiento y partícipe privilegiado del proceso de transposición: es el profesor quien recupera el conocimiento cotidiano y lo proyecta hacia el científico desde una propuesta académica, legitimándolo en las interacciones áulicas que genera. Por último, concluimos que enseñar la diversidad biológica lleva implicados una serie de saberes que deberían ser incluidos en las carreras de formación del profesorado en ciencias.

\section{Bibliografía}

Adúriz-Bravo, A. \& Erduran, S. (2003). La epistemología específica de la Biología como disciplina emergente y sus posibles contribuciones a la didáctica de la Biología. REB, 6(1), 9-13.

Acevedo Díaz, J. (2009). Conocimiento didáctico del contenido para la enseñanza de la naturaleza de la ciencia (I): el marco teórico. Revista Eureka, 6(1), 21-46.

Bermudez, G., De Longhi, A.L., Díaz, S. \& Gavidia, V. (en prensa). El concepto de diversidad biológica en libros de texto de la educación secundaria española. Enseñanza de las Ciencias.

Bermudez, G., García Capocasa, M.C., Battistón, L.V., Díaz, S. \& De Longhi, A.L. (2012). El conocimiento de las especies vegetales nativas de estudiantes de escuela media en Córdoba, Argentina. $V$ Congreso Internacional de Enseñanza de la Biología. 11-13 de octubre. Villa Giardino, Córdoba.

Bermudez, G. \& De Longhi, A.L. (2011). El conocimiento didáctico de contenidos biológicos de Ecología. En Flores, R.C. (Ed.). Experiencias Latinoamericanas en educación ambiental (pp. 19-35). Méjico: CECyTE-CAEIP.

Bermudez, G. \& De Longhi, A. (2008). La Educación Ambiental y la Ecología como ciencia. Una discusión necesaria para la enseñanza. REEC, 7(2), 275-297. 
Bio-grafía Escritos sobre la Biologia y su Enseñanza.

Edición Extra-Ordinaria. ISSN 2027-1034 P. p 996 - 1007

Memorias del VII Encuentro Nacional de Experiencias en la Enseñanza de la

Biología y la Educación Ambiental y 11 Congreso Nacional de Investigación en la Enseñanza de la Biología

Cardoso-Silva, C.B. \& de Oliveira, A.C. (2013). Como os livros didáticos de biologia abordam as diferentes formas de estimar a biodiversidade? Ciência \& Educação, 19(1), 169-180.

Cervini, R. (2003). Diferencias de resultados cognitivos y no-cognitivos entre estudiantes de escuelas públicas y privadas en la educación secundaria de Argentina: Un análisis multinivel. Education Policy Analysis Archives, 11(5). Disponible en: http://epaa.asu.edu/epaa/v11n6/.

Césere, S., Incatasciato, A., Pavese, S., Meehan, A. 2013. Uso paisajístico de plantas. Componentes naturales del paisaje. Córdoba: UNC. (En edición).

Díaz, S., Fargione, J., Chapin, F. III \& Tilman. D. (2006). Biodiversity loss threatens human well-being. PLoS Biology, 4(8), e277. doi:10.1371/journal.pbio.0040277.

Campos, C.M., Greco, S., Ciarlante, J.J., Balangione, M., Bender, J.B., Nates, J. \& Lindermann- Matthies, P. (2012). Students' familiarity and initial contact with species in the Monte desert (Medonza, Argentina). Journal of Arid Environments, $82,98-105$.

Chevallard, I. (1991). La Transposición Didáctica. Del saber sabio al saber enseñado. Buenos Aires: Aique.

Gómez Mendoza, M.A. (2005). La transposición didáctica: historia de un concepto. Revista Latinoamericana de Estudios Educativos, 1 (Julio - Diciembre), 83-115.

Menzel, S. \& Bögeholz, S. (2009). The loss of biodiversity as a challenge for sustainable development: how do pupils in Chile and Germany perceive dilemmas? Research in Science Education, 39, 429-447.

Nates, J., Campos, C. \& Lindermann-Matthies, P. (2010). Students' perception of plant and animal species: a case study from rural Argentina. Applied Environmental Education and Communication, 9, 131-141.

Schwarz, M.L., André, P., Sevegnani, L. (2012). Children's representations of the biological richness of the mata atlântica biome. Ciência \& Educação, 18(1), 155172.

Shulman, L. (1987). Knowledge and teaching: Foundations of the new reform. Harvard Educational Review, 57, 1-22.

Shulman, L. (1986). Those who understand: Knowledge growth in teaching. Educational Researcher, 15(2), 4-14. 
Bio-grafia Escritos sobre la Biologia y su Enseñanza.

Edición Extra-Ordinaria. ISSN 2027-1034 P.p 996 - 1007

Memorias del VII Encuentro Nacional de Experiencias en la Enseñanza de la

Biología y la Educación Ambiental y II Congreso Nacional de Investigación en la Enseñanza de la Biología

\section{Anexo 1}

Cuestionario para alumnos sobre distintos componentes y atributos de la biodiversidad.
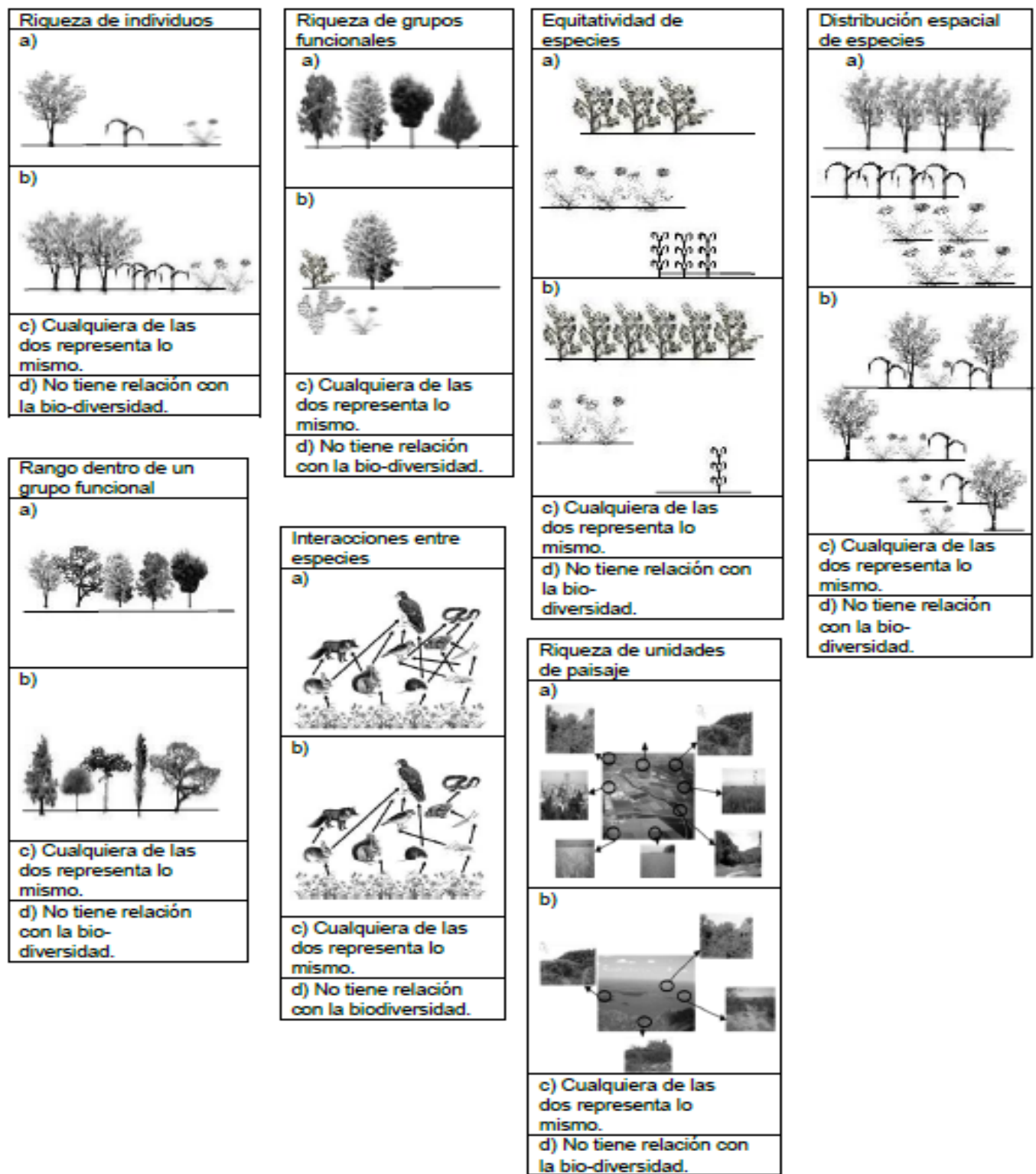